\title{
Fenomenología como método de investigación: Una opción para el profesional de enfermería
}

\author{
Fernando Trejo Martínez* \\ RESUMEN
}

El profesional de enfermería necesita métodos de estudio que le permitan observar al ser humano como un ente indivisible, singular y único en el mundo, que vive, siente y percibe de manera individual y propia. El objetivo de este artículo es analizar la fenomenología como método de investigación (una opción para el profesional de enfermería desde el punto de vista filosófico) y proporcionar elementos que le permitan realizar estudios de tipo cualitativo, ofreciendo una óptica diferente. Se explica cómo surge la fenomenología, sus preceptos, precursores, la visión que tuvieron para analizar las cosas (fenómenos) y el método de estudio que se desarrolló para enfocar su teoría y cómo ésta se fue adaptando a una nueva forma para llevar a cabo la investigación. Asimismo, se desarrolla este método para que los profesionales de la salud puedan comprender el comportamiento humano, aprovechando todos los recursos que se tienen para ofrecer un cuidado holístico.

Palabras clave: Fenomenología, método fenomenológico, investigación cualitativa, profesional de enfermería.

\section{Phenomenology as a research method: an option for the nurse}

\begin{abstract}
The nurse needs to study methods that allow you to see the human being as a indivisible, singular, unique in the world you live in, feel and perceive individually and own. The objective of this article is to analyze the phenomenology as a research method: an option for the nurse, from the philosophical point of view and provide elements that allow qualitative studies, offering a different perspective. It describes as phenomenology arises, its precepts, precursors, had the vision to analyze things (phenomena), and the method of study to focus on developing its ideology and how it was adapted to a new way to bring out research. Also this method is developed for health professionals to understand human behavior using all the resources they have to offer holistic care.
\end{abstract}

Key words: Phenomenology, phenomenological method, qualitative research, nurse.

\section{INTRODUCCIÓN}

$\mathrm{L}$ a fenomenología surgió como una necesidad de explicar la naturaleza de las cosas (fenómenos). Los primeros pensadores trataron de definir si era un método o una filosofía, ${ }^{1}$ dado que lejos de ser una secuencia de pasos, es un nuevo paradigma que observa y explica la ciencia para conocerla exactamente y, de esta forma, encontrar la verdad de los fenómenos. ${ }^{2}$

No obstante, lejos de dilucidar si se trataba de una estructuración de pensamiento para llegar a la verdad, se encontró que la representación de los fenómenos es una subjetividad del pensamiento; de esta manera, al intentar darle un sentido indiscutible encontraron que existen dos razonamientos: uno

\footnotetext{
* Maestría en Enfermería en Educación.

Instituto Nacional de Neurología y Neurocirugía.
}

Correspondencia: Fernando Trejo Martínez. Insurgentes Sur Núm. 3877, Col. La Fama, 14269, Delegación Tlalpan, México, D.F. Correo electrónico: fertre001@gmail.com

Este artículo puede ser consultado en versión completa en http://www.medigraphic.com/enfermerianeurologica 
precientífico y otro científico. El precientífico se refiere a aspectos del espíritu; el científico a los de las ciencias objetivas. Al mismo tiempo, se enfrentaron a otro problema de índole filosófica, pues al tratar de explicar los hechos, éstos tenían que ser verdaderos para ser científicos. El dilema filosófico consistió en darle carácter científico a la subjetividad del pensamiento; de esta manera, se permitiría rechazar los postulados del realismo empírico y establecer los fundamentos del positivismo, y por tanto, de lo científico. Sin embargo, para entender lo subjetivo del pensamiento no existía una estructura científica que definiera estos conceptos para hacerlos reales, por lo cual se juzgaron empíricos. ${ }^{3}$ Husserl, en este sentido, trató de explicar y fundamentar que la ciencia apela a las características psicológicas de la especie humana (en específico de su mente), y puso de ejemplo a las matemáticas; su fundamentación fue: analizar la estructura científica de la mente humana. En ese tiempo, se consideraba al cerebro como la mente; la conclusión, entonces, era que las leyes psíquicas se debían al funcionamiento del cerebro y, por tanto, resultaba un axioma netamente físico y tangible, por lo mismo considerado verdadero y científico.

Husserl argumentó, además, que existe en nosotros un "medio vital cotidiano"; también explicó cómo este mundo de vida funge constantemente de trasfondo con sus dimensiones valorativas prelógicas y funcionales para las verdades lógicas y teóricas; refiere así que todo conocimiento teórico es como un soporte vital y natural. Para Husserl, la fenomenología es la ciencia que trata de descubrir las estructuras esenciales de la conciencia. ${ }^{4}$

Heidegger, por otra parte, precisa que la fenomenología se enfatiza en la ciencia de los fenómenos; ésta consiste en "permitir ver lo que se muestra, tal como se muestra a sí mismo y en cuanto se muestra por sí mismo"; por consecuencia, es un fenómeno objetivo, por lo tanto verdadero y a su vez científico. ${ }^{5}$

El profesional de enfermería tiene que basar su cuidado en entender lo que la gente siente o piensa cuando está frente a un evento que desestabiliza su vida cotidiana. La fenomenología se dirige al estudio de la experiencia vivida respecto de una enfermedad o circunstancia por el propio protagonista de la experiencia ${ }^{6}$ y busca describir los significados de los fenómenos experimentados por los individuos a través del análisis de sus descripciones.

Numerosos son los estudios que evidencian el fracaso de las concepciones predeterminadas, fragmentadoras, selectivas y autoritarias de nuestra especial contribución al cuidado de la salud; esto ha llevado a numerosos profesionales de enfermería a reflexionar sobre diversas perspectivas. ${ }^{7} \mathrm{La}$ fenomenología permite brindar un cuidado más acorde a lo que la persona espera del profesional. Es la ciencia que considera cómo revelar y hacer visibles las realidades o cómo acceder a la variedad que profundiza toda realidad. Husserl la considera como una filosofía, un enfoque y un método; del mismo modo enfatiza en la intuición reflexiva para describir la experiencia tal como se vive y, desde su punto de vista, todas las distinciones de nuestra experiencia deben carecer de presuposiciones y prejuicios; en cambio, se deben buscar los fundamentos teóricos que permitan crear una base segura para describir la experiencia y conseguir la realidad del mundo tal y como es. ${ }^{8}$

Heidegger refiere que es una interpretación, aclaración explicativa del sentido del ser, un mundo sociohistórico donde la dimensión fundamental de toda conciencia humana es histórica y sociocultural y se expresa por medio del lenguaje. ${ }^{9}$ Asimismo, menciona que las personas son un ser en el mundo, pero no sólo un mundo físico: éste incluye sus relaciones con las demás personas, además es un mundo que él construye y modifica, pero sobre todo que se encuentra inmerso en él, así como en el estudio de su mundo sugiere la forma de interpretarlo y vivirlo. ${ }^{10}$ Éstas son situaciones que el profesional de enfermería tiene que evaluar, ya que muchas de las enfermedades se originan de la relación entre la persona y el medio.

Esto fundamenta las bases para entender a las personas a quienes se proporciona el cuidado, para saber cuáles son sus reacciones, sentimientos y experiencias, y así poder brindar un cuidado real y específico de las necesidades que manifiesten. Tal como lo menciona Watson, el cuidado es una relación intersubjetiva ${ }^{11}$ en la cual se trata de entender al sujeto como un igual; significa proporcionar un cuidado humano.

Resumiendo, conforme lo que explican tanto Husserl como Heidegger se define a la fenomenología como el estudio de los fenómenos (o experiencias) tal como se presentan y la manera en que se vive por las propias personas.

Enfermería, como disciplina humanística, necesita métodos de investigación que la acerquen a su objeto de estudio (el cuidado de la persona). Pero estos métodos deben ayudarnos a ver a cada individuo como a un ser con respuestas y sentimientos propios, y que sólo él los experimenta de manera única. Es fundamental que no se generalice sobre la conducta humana, ya que es la persona misma quien construye su mundo y lo vive de acuerdo a su propia percepción de la realidad. Esto hace que tenga sus propias respuestas a diversas situaciones, incluidas las enfermedades, respuestas que también son influidas por una gran cantidad de factores. Los métodos de investigación cualitativos ofrecen una gama de posibilidades para poder explorar este tipo de contextos, además de proporcionar una visión que se adapte mejor al ambiente que el profesional de enfermería necesita examinar. Asimismo, los resultados y el análisis de los mismos 
brindan información que vincula o integra resultados cuantitativos que no son capaces de reflejar, porque no es lo mismo un porcentaje que experimentar una enfermedad, o un dato estadístico que la incertidumbre de pensar si la cirugía aliviará al familiar o no.

Como se ha descrito anteriormente, no sólo es una forma distinta de investigar, sino que se trata de un cambio de paradigma, de cómo puede el enfermero cambiar la visión de nuestro entorno profesional, basándose en el estudio de los fenómenos que determinan la conducta humana. De esta manera, el entender mejor a la persona, nos va a servir para proporcionar un cuidado acorde a lo que la sociedad espera del profesional de enfermería, considerando a la disciplina como una ciencia humanística que estudia no sólo a la persona sino también a su entorno.

La finalidad de este ensayo es exponer los motivos por los cuales la fenomenología es un método ideal para investigar; la misma refleja una filosofía y un paradigma y nos ofrece una enorme gama de posibilidades para explorar la conducta humana.

Las etapas del método fenomenológico. Para la realización de estos estudios de corte cualitativo se recurre a las siguientes etapas: descriptiva, estructural y de discusión.

\section{Etapa descriptiva}

El objetivo de esta etapa es lograr una descripción del fenómeno de estudio, lo más completa y no prejuiciadamente posible, que refleje la realidad vivida por la persona, su mundo, su situación en la forma más auténtica. Consta de tres pasos:

1) Elección de la técnica o procedimiento. Aquí se pretende lograr la descripción lo más exacta posible utilizando varios procedimientos:

La observación directa o participativa, en los eventos vivos (se toman notas, recogiendo datos, etcétera), pero siempre tratando de no alterarlos con la presencia del investigador.

La entrevista coloquial o dialógica, con los sujetos de estudio con anterioridad a esta etapa; se deben de estructurar en partes esenciales para obtener la máxima participación de los entrevistados y lograr la mayor profundidad de lo vivido por el sujeto.

La encuesta o el cuestionario deben ser parcialmente estructurados, abiertos y flexibles, de tal manera que se adapte a la singularidad de cada sujeto en particular.

El autorreportaje. Éste se lleva a cabo a partir de una guía que señale las áreas o preguntas fundamentales a ser tratadas. Estos procedimientos no son excluyentes, sino que más bien se integran a la práctica.

2) La aplicación de la técnica o procedimiento seleccionado toma en cuenta lo siguiente: que nuestra percepción aprende estructuras significativas; generalmente vemos lo que esperamos ver; los datos son casi siempre para una u otra hipótesis; nunca observamos todo lo que podríamos haber observado, y la observación es siempre selectiva y siempre hay una correlación funcional entre la teoría y los datos: las teorías influyen en la determinación de los datos, tanto como éstos en el establecimiento de las teorías.

No obstante, la puesta en práctica de estas consideraciones, como cualquier intento de una objetividad rigurosa, es muy difícil; imposible de realizar con tanta pureza, aunque para lograrlo se requiere de una considerable aptitud, entrenamiento y autocrítica.

3) Elaboración de la descripción protocolar: un fenómeno bien observado y registrado no será difícil de describir con características de autenticidad, cumpliendo con los siguientes presupuestos: que reflejen el fenómeno o la realidad tal como se presentó; que sea lo más completa posible y no omita nada que pudiera tener relevancia para el estudio; que no contenga elementos "proyectados por el observador", ideas suyas, teorías consagradas, prejuicios propios o hipótesis plausibles; que recoja el fenómeno descrito en su contexto natural, en su situación particular y el mundo propio en que se presenta, y que la descripción efectuada aparezca como una verdadera "ingenuidad disciplinada".

\section{ETAPA ESTRUCTURAL}

En esta etapa, el trabajo central es el estudio de las descripciones contenidas en los protocolos; éstos están constituidos de varios pasos entrelazados, y aunque la mente humana no respeta secuencias tan estrictas, ya que en su actividad cognoscitiva se adelanta o vuelve atrás con gran rapidez y agilidad para dar sentido a cada elemento o aspecto, sin detenerse en cada uno los ve por separado, de acuerdo a la prioridad temporal de la actividad en que pone énfasis; es recomendable seguir la secuencia de éstos para tener un mejor análisis de los fenómenos estudiados. Los pasos son los siguientes:

Primer paso: Lectura general de la descripción de cada protocolo.

Segundo paso: Delimitación de las unidades temáticas naturales.

Tercer paso: Determinación del tema central que domina cada unidad temática.

Cuarto paso: Expresión del tema central en lenguaje científico. 
Quinto paso: Integración de todos los temas centrales en una unidad descriptiva.

Sexto paso: Integración de todas las estructuras particulares en una estructura general.

Séptimo paso: Entrevista final con los sujetos de estudio. ${ }^{12}$

\section{ETAPA DE DISCUSIÓN DE LOS RESULTADOS}

En esta etapa se intenta relacionar los resultados obtenidos de la investigación con las conclusiones o hallazgos de otros investigadores para compararlos, contraponerlos o complementarlos, y entender mejor las posibles diferencias o similitudes. De este modo, es posible llegar a una mayor integración y a un enriquecimiento del "cuerpo de conocimientos" del área estudiada.

Como se podrá observar, la fenomenología, lejos de ser un método de estudio, es una filosofía para entender el verdadero sentido de los fenómenos, pero con una secuencia de ideas y pasos que le dan rigurosidad científica.

\section{BIBLIOGRAFÍA}

1. Hegel G. La fenomenología del espíritu. Fondo de Cultura Económica, México; 1966: 6.

2. Husserl E. Invitación a la fenomenología. Barcelona: Paidós; 1998: 13-23.

3. Martínez M. El comportamiento humano. $2^{a}$ Edición. México: Trillas; 1996: 167-169.

4. Husserl. Op. cit., 10

5. Heidegger M. Introducción a la fenomenología de la religión. México: Fondo de Cultura Económica; 2006: 233-256.

6. Palacios-Ceña, D. Corral, I. Fundamentos y desarrollo de un protocolo de investigación fenomenológica en enfermería. Revista de Enfermería. Intensiva. 2010 Vol. $21 \mathrm{~N}^{\circ} 2$ pág. 68-73.

7. Rivera MS, Herrera LM. Fundamentos fenomenológicos para un cuidado comprensivo de enfermería. Texto Contexto Enfermería, Florianópolis 2006; 16: 158-63.

8. Solano MC. Fenomenología-hermenéutica y enfermería. Cultura de los Cuidados 2006; 19: 5.

9. Ibíd., 6

10. Castillo, E. La fenomenología interpretativa como alternativa apropiada para estudiar los fenómenos humanos. Investigación y Educación en Enfermería. Marzo 2000. Vol $13 \mathrm{~N}^{\circ} 1$.

11. Watson, J. Nursing: Human science and human care. Editado por la National League for Nursing. New York 1988 pág. 25-52.

12. Martínez M. Op. cit., 171-178. 